\title{
A Shadow-Overlapping Algorithm for Estimating Building Heights From VHR Satellite Images
}

\author{
Nada Kadhim $^{\circledR}$, Member, IEEE, and Monjur Mourshed ${ }^{\circledR}$, Member, IEEE
}

\begin{abstract}
Building height is a key geometric attribute for generating 3-D building models. We propose a novel four-stage approach for automated estimation of building heights from their shadows in very high resolution (VHR) multispectral images. First, a building's actual shadow regions are detected by applying ratio-band algorithm to the VHR image. Second, 2-D building footprint geometries are identified using graph theory and morphological fuzzy processing techniques. Third, artificial shadow regions are simulated using the identified building footprint and solar information in the image metadata at predefined height increments. Finally, the difference between the actual and simulated shadow regions at every height increment is computed using Jaccard similarity coefficient. The estimated building height corresponds to the height of the simulated shadow region that resulted in the maximum value for Jaccard index. The algorithm is tested on seven urban sites in Cardiff, U.K. with various levels of morphological complexity. Our method outperforms the past attempts, and the mean error is reduced by at least $21 \%$.
\end{abstract}

Index Terms-Building detection, building height estimation, Jaccard index, morphological dilation, region fitting, shadow detection, very high resolution (VHR) satellite imagery.

\section{INTRODUCTION}

G EOMETRY identification of buildings and subsequent (3-D) modeling play an important role in a range of urban applications-from urban energy and environmental analysis [1] and the estimation of renewable energy potential [2] to data-centric operation and management of smart and resilient cities [3]. Building height $\left(H_{\mathrm{B}}\right)$ is one of the key geometric parameters that is used to transform the (2-D) footprint area into a 3-D model. Manually obtaining $H_{\mathrm{B}}$ from a large number of buildings for urban-scale 3-D modeling and analysis is resource intensive. The difficulty and cost involved in $H_{\mathrm{B}}$ estimation also create a barrier to the use and deployment of advanced modeling, analysis, and management of the built environment for most, if not all cities and countries. Finding an efficient and cost-effective way to estimate $H_{\mathrm{B}}$ is, therefore, of paramount importance.

Manuscript received June 13, 2017; revised August 18, 2017 and October 3, 2017; accepted October 9, 2017. Date of publication December 4, 2017; date of current version December 27, 2017. This work was in part by the Higher Committee for Education Development, Office of the Prime Minister, Baghdad, Iraq, and in part by the Cardiff University Open Access Support Team University Library Service, Cardiff University, U.K. (Corresponding author: Nada Kadhim.)

N. Kadhim is with the School of Engineering, Cardiff University, Cardiff CF24 3AA, U.K., and also with the Department of Civil Engineering, University of Diyala, Baqubah, Iraq (e-mail: mohammedsalihnm@cardiff.ac.uk).

M. Mourshed is with the School of Engineering, Cardiff University, Cardiff CF24 3AA, U.K. (e-mail: mourshedm@ cardiff.ac.uk).

Color versions of one or more of the figures in this letter are available online at http://ieeexplore.ieee.org.

Digital Object Identifier 10.1109/LGRS.2017.2762424
A common factor in the extraction of $H_{\mathrm{B}}$ approaches based on remotely sensed elevation data is that they require sophisticated data calibration and processing to obtain a reliable digital surface model (DSM). Although studies have shown the utility and usefulness of elevation data for extracting $H_{\mathrm{B}}$, their implementation typically requires the use of additional data and often multiple images from different angles to obtain a satisfactory view of building size and shape [4]. Another feature of elevation data-based $H_{\mathrm{B}}$ extraction is the need for data preprocessing because of point cloud sparsity and data misalignment [5]. As an alternative to costly data acquisition and processing, several studies have developed methods for obtaining $H_{\mathrm{B}}$ from one data source, such as satellite images utilizing the shadows cast by buildings.

This letter presents an original approach, termed the shadow-overlapping algorithm, $A_{\mathrm{SO}}$, for the automated estimation of $H_{\mathrm{B}}$ from monocular very high resolution (VHR) multispectral pan-sharpened satellite images. The contributions of this paper are threefold: 1) the generation of artificial shadows, $S_{\mathrm{Ar}}$ from a simulation of the actual shadows, $S_{\mathrm{Ac}}$ of the buildings in the image space; 2) the solution to the issue of overlapping shadows of the multiple buildings; and 3) the development of an algorithm by combining 1) and 2) for the automated estimation of $H_{\mathrm{B}}$ by identifying the optimal height value for the given building.

The rest of this letter is structured as follows. Previous work on $H_{\mathrm{B}}$ estimation is reviewed in Section II. Our approach and the simulation process are described in Section III. Experimental results are discussed in Section IV, while Section V provides concluding remarks and directions of the future work.

\section{Previous Work}

The first task in shadow-based $H_{\mathrm{B}}$ estimation is the extraction of shadow regions from VHR satellite images. In this respect, [6] reported two widely used techniques: ratio-band and Graph-Cut partitioning via kernel mapping, with overall accuracies of $85 \%$ and $79 \%$, based on two performance metrics, $F_{1}$ score (harmonic mean of precision and recall) and probabilistic Rand index, respectively. A semiautomatic approach was proposed in [7] to estimate $H_{\mathrm{B}}$ from a single satellite image by manually adjusting the height of a simulated building and then matching the projected shadow with the actual. In contrast, [8] used volumetric shadow analysis to automatically extract $H_{\mathrm{B}}$, which is primarily designed for buildings with full scenes of their bases and rooftops, including the sides of the building. Reference [9] also matched shadow regions but estimated $H_{\mathrm{B}}$ using simple triangulation. Estima- 


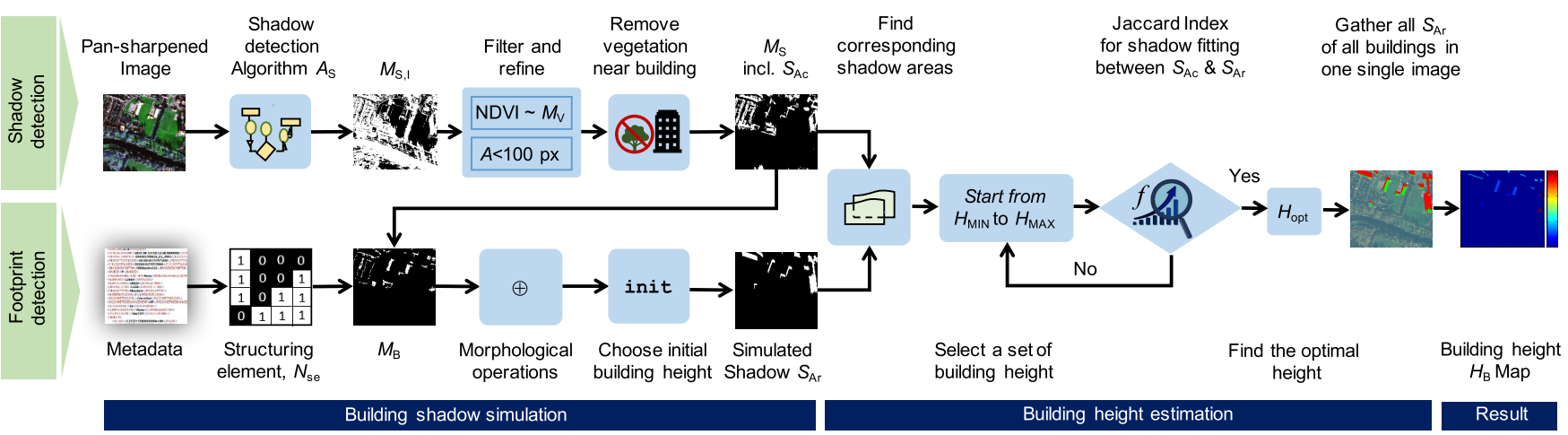

Fig. 1. Framework of the shadow-overlapping algorithm for building height estimation.

tion accuracy in this approach is dependent on the quality of the segmented rooftop polygons.

Shao et al. [10] used a classification approach to detect shadows and characterized the relationship between buildings and their shadows to estimate $H_{\mathrm{B}}$, with overall classification accuracy of $87 \%$ evaluated by a confusion matrix. The method overestimates the shadow lengths of buildings, resulting in large errors in calculated $H_{\mathrm{B}}$. In [11], the number of a building's storeys as well as its length and shape were inferred based on the identification of shadow areas. In the absence of detailed validation, it appears that the empirical nature of the rule-based classification may only be practical for the presented cases. In [12], $H_{\mathrm{B}}$ was estimated from the extracted shadow regions based on the sun-satellite geometry relationship using three different approaches: ratio, examplebased, and rule-based with mean error in estimated $H_{\mathrm{B}}$ of 0.67 , 1.51 and $0.96 \mathrm{~m}$, respectively. The method does not consider overlapping shadow regions caused by other buildings and vegetation, limiting its applicability in dense urban areas. Reference [13] applied a semiautomated triangular analysis of shadow geometry to estimate $H_{\mathrm{B}}$, which is computationally expensive and may restrict its use if there are many buildings in the scene.

More recently, [14] estimated $H_{\mathrm{B}}$ from Google Earth $^{1}$ images by first calculating the ratio of $H_{\mathrm{B}}$ to the shadow length of known buildings, and thereafter utilizing the identified shadow-length ratio to obtain heights of other buildings with unknown heights. The approach sits somewhere between direct and indirect approaches as some field measurements are required. Reference [15] developed a custom filter for enhancing shadows and reducing the spectral heterogeneity of the regions of interest (ROIs) to form an optimized contour model for estimating $H_{\mathrm{B}}$ using a shadow length and a solar elevation angle. However, the presented approach is not tailored to detect the ROIs of objects with spectral dissimilarity. $F_{1}$ score evaluation results illustrate a large aggregate height variance $(4.13 \mathrm{~m})$ due to the underestimation of building shadow lengths. In the studies reviewed here, the lowest and highest root-mean-square errors (RMSEs) were found to be $0.98 \mathrm{~m} \mathrm{[14]} \mathrm{and} 22.66 \mathrm{~m}$ [15], respectively. Error estimates of all reviewed literature are provided in Table II for comparison against this letter.

\footnotetext{
${ }^{1}$ Google Earth. https://earth.google.com.
}

\section{Proposed Method}

The framework of the shadow-overlapping algorithm, $A_{\mathrm{SO}}$, is shown in Fig. 1, and the steps are discussed as follows.

\section{A. Identification of the Building Shadow Mask}

To reliably extract the actual shadows of buildings, $S_{\mathrm{Ac}}$, we applied a refined version of our previously developed shadow detection algorithm, $A_{S}$ [6], as proposed by Rüfenacht et al. [16]. Candidate shadow regions are obtained by applying a nonlinear mapping function to the extracted dark pixels from both the visible, $V$, and near infrared, NIR, bands using

$$
f(x)=\frac{1}{1+e^{-\alpha\left(1-x^{\frac{1}{\gamma}}-\beta\right)}}
$$

where $\alpha, \beta$, and $\gamma$ are the parameters to control the sigmoid function. Each candidate dark pixel in $V$ is multiplied with its counterpart in NIR to obtain the candidate shadow image $D$. In cases where $V$ and $N I R$ pixel values are dark, the calculation of image ratio $(T=V / N I R)$ can affect shadow detection. Both $D$ and $T$ are in the range $[0,1]$. The final shadow candidate image $I_{\mathrm{S}}$ is calculated ${ }^{2}$ using

$$
I_{\mathrm{S}}=(1-D)(1-T) \text {. }
$$

We then applied image thresholding to $I_{\mathrm{S}}$ to obtain the initial shadow mask, $M_{\mathrm{S}, \mathrm{I}}$, where the influence of noise is reduced by computing the histogram ${ }^{3}$ of $I_{\mathrm{S}}$. Although noise reduction from $M_{\mathrm{S}, \mathrm{I}}$ was conducted to obtain an accurate binary image of the initial shadow mask, small dark areas of nonshadow pixels remained. We, therefore, proposed a morphological filter to remove all small objects, i.e., the nonshadow pixels. To keep only the areas of building shadows, the process of elimination of small objects is controlled by removing the areas $<100$ pixels [6]. In this letter, $A_{S}$ is improved further to detect and eliminate the vegetation cover by applying normalized difference vegetation index. A binary vegetation mask $M_{\mathrm{V}}$ is extracted using automatic histogram thresholding from [18]. Thereafter, we subtracted $M_{\mathrm{V}}$ from $M_{\mathrm{S}, \mathrm{I}}$ to obtain the final building shadow mask $M_{\mathrm{S}}$. To eliminate shadows from vegetation canopies near buildings,

\footnotetext{
${ }^{2}$ Equations 1 and 2 are discussed further in [16].

${ }^{3}$ The number of histogram bins is selected based on Sturges' rule [17].
} 
we applied a probabilistic fuzzy landscape approach from [19] that applies low and high thresholds to the membership values of the generated fuzzy landscapes.

\section{B. Generation of the Artificial Shadow}

A binary image of the building footprint or region is required to simulate its shadows for estimating $H_{\mathrm{B}}$. To do so, we automatically detected the building footprints using the graph theory-based approach proposed by Ok [19]. We used the first level of partitioning in which building footprints are determined by iterative Graph-Cut performed in twolabel partitioning comprising the background and foreground. As this letter is one of the first use of the images from new satellite sensors, WorldView-3 (WV3), for object detection, we conducted further refinements to the approach, in particular on the adjustment of the thresholding values, fuzzy landscape parameters, and the geometry of the extracted building regions. The mask of buildings $M_{\mathrm{B}}$ was then obtained where the building footprints are accurately separated from their background. Thereafter, based on a flat terrain assumption, ${ }^{4}$ we utilized solar information ${ }^{5}$ in the image metadata to generate a flat linear morphological opening, the line structuring element, which is symmetric with respect to the neighborhood center. The direction of the sun illumination is maintained using the solar azimuth angle $[\lambda$, as per 3$]$ to determine the length of the smallest connected single edge segment $L$, using 4

$$
\begin{aligned}
\lambda & =A z-\pi / 2 \\
L & =\frac{H_{\mathrm{T}}^{\max }}{\tan \phi R_{\mathrm{img}}}
\end{aligned}
$$

where $H_{\mathrm{T}}^{\max }$ is the maximum height threshold for the buildings that cast shadows, $A z$ is the azimuth, $\phi$ is the solar elevation angle $E l$, and $R_{\mathrm{img}}$ is the image resolution. After creating the line structuring element, we investigated the connected components of buildings in $M_{\mathrm{B}}$ and their shadows in $M_{\mathrm{S}}$ to label each component with an eight-connected neighborhood. The connected components are then extracted with a unique label for creating a mask of their perimeter pixels $M_{\mathrm{Bp}}$, using

$$
M_{\mathrm{Bp}}=\left(M_{\mathrm{Bc}} \otimes O_{3 \times 3}\right) \cap M_{\mathrm{B}}
$$

where $M_{\mathrm{Bc}}$ is the complement of the building object equation (6), and $O_{3 \times 3}$ is a matrix of ones. $\otimes$ and $\cap$ denote morphological dilation and pixel value intersection, respectively, using

$$
M_{\mathrm{Bc}}=1-M_{\mathrm{B}} .
$$

Once $M_{\mathrm{Bp}}$ is identified, $A_{\mathrm{SO}}$ then simulates $S_{\mathrm{Ac}}$ in the opposite direction of the solar illumination to generate new regions of building shadows $S_{\text {sim }}^{\max }$, using

$$
S_{\mathrm{sim}}^{\max }=\left(M_{\mathrm{Bp}} \otimes N_{\mathrm{se}}\right) \cap M_{\mathrm{Bc}}
$$

\footnotetext{
${ }^{4}$ Acute solar elevation angles $\left(\phi<30^{\circ}\right)$ lead to large cast shadows [19].

${ }^{5}$ Contains date, time, solar illumination angles (azimuth and elevation), and the angles of solar projection, i.e., the viewing geometry during image acquisition. The images are supplied georeferenced and orthorectified to Earth surface coordinate system (data: UTM).
}

where $N_{\text {se }}$ is the neighborhood associated with the structuring element (se). $S_{\mathrm{Ar}}$ for each building region was then identified using

$$
S_{\mathrm{Ar}}=S_{\mathrm{sim}}^{\max } \cap M_{\mathrm{S}}
$$

Because of shadows cast by adjacent and connected buildings, some shadow areas in $S_{\mathrm{Ac}}$ may not have corresponding shadows in $S_{\mathrm{Ar}}$. Matching between both shadows is, therefore, evaluated using $F_{1}$ score as $A_{\text {SO }}$ keeps tracing the trail of each $S_{\mathrm{Ac}}$ in the shadow mask $M_{\mathrm{S}}$ to fit each $S_{\mathrm{Ar}}$ with the corresponding $S_{\mathrm{Ac}}$ in $M_{\mathrm{S}}$.

\section{Estimation of Building Heights}

To estimate the heights of the buildings from a single VHR satellite image, we develop the shadow-fit function $f_{\mathrm{sf}}$ based on Jaccard index $(J I)$, which yields $A_{\mathrm{SO}}$ to compute the fitting connected components over the pixels between $S_{\mathrm{Ac}}$ and $S_{\mathrm{Ar}}$ regions. The estimated values of $H_{\mathrm{B}}$ are extracted depending on the optimal height of a specific building using a set of $H_{\mathrm{B}}$, solar angles $A z$ and $E l$, the number of buildings in $M_{\mathrm{B}}, R_{\mathrm{img}}$, $M_{\mathrm{B}}$, and $M_{\mathrm{S}}$ into $f_{\mathrm{sf}}$. We measure how fit and similar the two shadow regions are by Jaccard similarity coefficient using

$$
J I=\left|S_{\mathrm{Ac}} \cap S_{\mathrm{Ar}}\right| /\left|S_{\mathrm{Ac}} \cup S_{\mathrm{Ar}}\right| .
$$

$J I$ computes the size of the intersection $\left(S_{\mathrm{Ac}}\right.$ and $\left.S_{\mathrm{Ar}}\right)$ divided by the size of the union of the two regions. The computation of the overlap by $J I$ between $S_{\mathrm{Ac}}$ and $S_{\mathrm{Ar}}$ is iterated until $A_{\text {SO }}$ finds the maximal index of fitting the two shadow regions, which approaches to 1 . The algorithm then extracts the highest index, which will represent the value of the optimal height $H_{\text {opt }}{ }^{6}$ of a given building. The final shadow region is simulated within the image space using $H_{\mathrm{opt}}, A z, E l$, $R_{\mathrm{img}}$, and a given building footprint in the $M_{\mathrm{B}}$ into another developed function $f_{\text {ssim }}$ to visualize and combine all outputs of the $S_{\mathrm{Ar}}$ regions into a single image. $A_{\mathrm{SO}}$ performance is evaluated using the RMSE measure, as in 10 , where $y_{i}$ and $\hat{y}_{i}$ are actual and predicted values, respectively, using

$$
\operatorname{RMSE}=\sqrt{\frac{1}{n} \sum_{i=1}^{n}\left(y_{i}-\hat{y}_{i}\right)^{2} .}
$$

\section{EXPERIMENTAL RESUlTS AND Discussion}

\section{A. Input Data Set}

The test images ${ }^{7}$ used in this paper contain seven orthorectified and pansharpened images, obtained from one of the latest satellites, VHR WV3 with a ground sampling distance of $40 \mathrm{~cm}$, as shown in the first column of Fig. 2. The images include four multispectral bands (B, G, R, and NIR) with a radiometric resolution of 16 bits per band. The WV3 images were taken with solar elevation $(E l)$ and azimuth $(A z)$ angles of $16.3^{\circ}$ and $173.2^{\circ}$, respectively, and with a maximum

\footnotetext{
${ }^{6}$ In this letter, the shadow regions are not obscured by their buildings ove the test images, because there is no significant difference between the azimuth and elevation angles of both the sun and the satellite. Hence, the correction of the building lengths derived from the shadow regions was not necessary.

${ }^{7}$ Source images can be downloaded from IEEE Xplore Digital Library.
} 


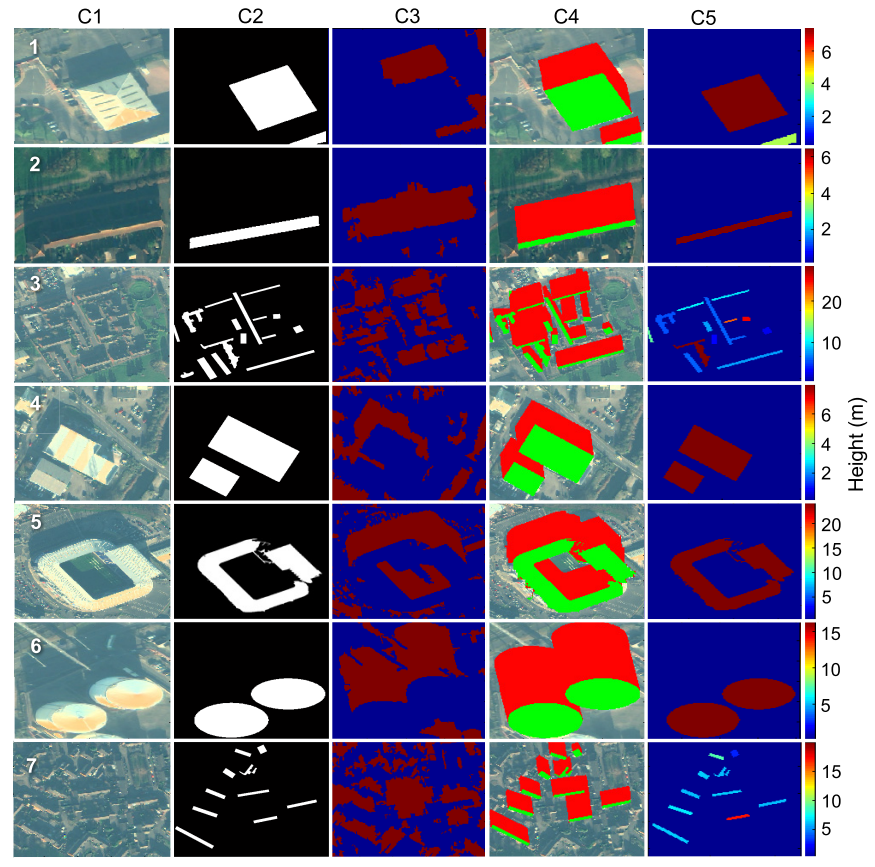

Fig. 2. Estimated values of building heights. C1: test images (\#1-7). C2: detected building footprints for test images. C3: extracted shadow regions for detected buildings. C4: artificial shadow regions for detected buildings. C5: colormaps of estimated building heights.

off-nadir angle of $27.6^{\circ}$ in which the cast shadow lengths were visible within the selected image. All test images are chosen to evaluate the performance of the new approach over diverse urban landscape scenes, which include different geometries in buildings in challenging environmental conditions. Moreover, we used VHR WV3 images to evaluate the usage of new satellite images in urban applications, as well as to compare the performance of the proposed approach to the existing methods for estimating building heights. The performance of our approach is evaluated using the reference building height data from Ordnance Survey (OS) ${ }^{8}$ MasterMap topography layer. Building heights of the corresponding urban areas of the test images in the OS reference data are used to compare results with the actual. The experiments were performed on an Intel i7 personal computer at $3.40 \mathrm{GHz}$ and 16-GB RAM.

\section{B. Results and Discussion}

Results are given in Fig. 2 and validation outputs in Table I. Algorithmic accuracy was measured using precision, recall, and $F_{1}$ score, which are presented in Fig. 3. The overall accuracy of our approach is compared against previous works on image-based building height estimation in Table II.

The results in Fig. 2 are promising considering the complex building characteristics of the test images, i.e., the variations in geometry, roof color, orientation, and challenging illumination conditions. As expected, the algorithm performs very well for standalone buildings of regular shape. Zero error was found

\footnotetext{
${ }^{8}$ The U.K. government agency is responsible for the official, definitive topographic survey and mapping of Great Britain. Building heights in OS MasterMap are automatically derived from digital terrain and surface models. Further details on their creation can be found here: https://goo.gl/ohws6c.
}

TABLE I

Estimated Building Heights Using the Proposed Algorithm

\begin{tabular}{|c|c|c|c|c|c|c|c|c|}
\hline \multirow[t]{2}{*}{ No. } & \multirow{2}{*}{$\begin{array}{l}\text { Size: } \mathrm{W} \times \mathrm{H} \\
(\mathrm{m})\end{array}$} & \multirow{2}{*}{$\begin{array}{l}T_{E}{ }^{*} \\
(\mathrm{~s})\end{array}$} & \multicolumn{3}{|c|}{ Building } & \multirow{2}{*}{$\begin{array}{l}H_{\mathrm{A}} \\
(\mathrm{m})\end{array}$} & \multirow{2}{*}{$\begin{array}{l}H_{\mathrm{E}} \\
(\mathrm{m})\end{array}$} & \multirow{2}{*}{$\begin{array}{l}\text { Error } \\
(\mathrm{m})\end{array}$} \\
\hline & & & Type* & Characteristics & Nos. & & & \\
\hline 1 & $85.6 \times 99.6$ & 6.76 & $\mathrm{C}$ & Detached & 1 & 7.5 & 7.5 & \\
\hline 2 & $72.4 \times 113.6$ & 3.36 & $\mathrm{R}$ & Connected & 1 & 6.1 & 6.5 & 0.4 \\
\hline \multirow[t]{6}{*}{3} & $201.2 \times 208.8$ & 132.36 & $\mathrm{R}$ & Mixed size & 6 & 7.75 & 7.5 & -0.25 \\
\hline & & & & & & 6.05 & 5.0 & -1.05 \\
\hline & & & & & & 9.5 & 10.5 & 1.0 \\
\hline & & & & & & 10.3 & 10.0 & -0.3 \\
\hline & & & & & & 7.3 & 7.5 & 0.2 \\
\hline & & & & & & 6.7 & 6.0 & -0.7 \\
\hline \multirow[t]{2}{*}{4} & $162.4 \times 132.4$ & 18.44 & I & Mixed size & 2 & 8.1 & 8.0 & -0.1 \\
\hline & & & & & & 5.45 & 8.0 & 2.55 \\
\hline 5 & $283.2 \times 365.6$ & 112.53 & $\mathrm{~S}$ & Mixed geometry & 1 & 24.1 & 24.5 & 0.4 \\
\hline \multirow[t]{2}{*}{6} & $81.2 \times 117.2$ & 6.29 & I & Mixed geometry & 2 & 17.5 & 17.0 & -0.5 \\
\hline & & & & & & 17.4 & 16.0 & -1.4 \\
\hline \multirow[t]{6}{*}{7} & $192.4 \times 172.4$ & 89.99 & $\mathrm{R}$ & Mixed size & 5 & 6.8 & 6.5 & -0.3 \\
\hline & & & & & & 6.7 & 6.5 & -0.2 \\
\hline & & & & & & 6.8 & 5.5 & -1.3 \\
\hline & & & & & & 6.8 & 6.0 & -0.8 \\
\hline & & & & & & 6.7 & 6.5 & -0.2 \\
\hline & & & & & & & MAE & 0.65 \\
\hline
\end{tabular}

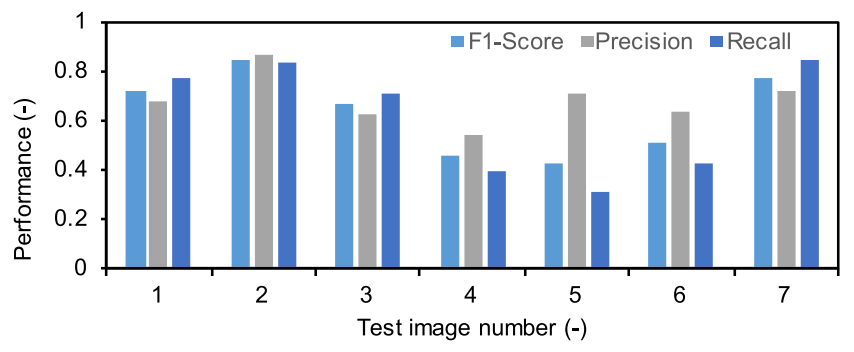

Fig. 3. Performance of the proposed shadow overlapping algorithm.

TABLE II

Algorithm Performance Against Previous Works

\begin{tabular}{|c|c|l|c|c|}
\hline Ref. & Year & VHR satellite imagery source & RMSE & Mean error $(\mathrm{m})$ \\
\hline$[7]$ & 2007 & Panchromatic IKONOS & 1.86 & 1.34 \\
\hline$[10]$ & 2011 & Panchromatic IKONOS & 12.99 & - \\
\hline$[9]$ & 2012 & QuickBird & 1.38 & 1.14 \\
\hline$[8]$ & 2013 & Panchromatic IKONOS & 1.34 & - \\
& & QuickBird & 1.71 & - \\
& & KOMPSAT2 & 1.67 & - \\
& & WorldView1 (WV1) & 1.88 & - \\
\hline$[14]$ & 2016 & Google Earth & 0.98 & 0.82 \\
\hline$[15]$ & 2016 & Google Earth & 22.66 & - \\
\hline This paper & - & WorldView3 (WV3) & 1.22 & 0.65 \\
\hline
\end{tabular}

for test image \#1, which is a detached commercial building. The algorithm underestimated the heights for mixed-geometry and mixed-size buildings in \#6 and \#7, respectively, by a small margin. The mean absolute error for all images was $0.65 \mathrm{~m}$, demonstrating the robustness of the algorithm.

One of the reasons for the success of our approach in challenging urban conditions is that in addition to applying a thresholding scheme to filter out the building from the background, our algorithm is also able to mitigate the issue of the overlapping shadow of two buildings. When the simulated shadow of a building overlaps the shadow of another, $J I$ is set to zero to avoid erroneous estimation of the building height. The performance appears to be affected by the presence of vegetation within the shadow region, as can be seen in image \#4. Two buildings are separated by a narrow gap. The shadow of the smaller building gets blocked by the larger building on the north. The estimation error for the larger 
building was $0.1 \mathrm{~m}(\approx 1 \%)$, while the error for the smaller building was $2.55 \mathrm{~m}(\approx 47 \%)$. The situation was exacerbated by the presence of tall vegetation on the northeastern side of the smaller building, preventing the accurate filtering of the shadow.

The Graph-Cut segmentation method [19] used for detecting building footprint worked well, except for image \#6, in which the side of the oil storage silo and the roof were of the same color. The shape of the building footprint was, therefore, larger than the actual [see Fig. 2 (C2)]. However, the shadows were unaffected and the overestimated footprint did not have a noticeable effect on the estimation of the building height. On the other hand, in cases where there are many shallow buildings such as images \#3 and \#7, the presence of darker pavements and roads can affect the performance of the algorithm. One possible reason is that the spectral reflectance of some nonshadow dark objects, such as roads and building roofs, are nearly identical to each other or to their background, resulting in dark objects being identified as shadow regions. In addition, some adjacent buildings occlude the shadow cast by other buildings. As a result, the length of the shadow region appears longer than its actual length if the shadow regions are combined with other shadow regions cast by other objects or buildings. In contrast, they will appear shorter if some parts of the shadow region are obstructed because of the adjacent buildings. Our approach mitigated this issue with the help of morphological postprocessing and thresholding in the simulation process. In addition, the approach removed all shadow pixels of nonbuilding objects, such as walls, cars, and utility pole that are independent of the building shadow.

Although satellite test images present diverse building attributes from different areas, the results demonstrate the effectiveness of our approach. The comparison of accuracy between the new and past approaches in Table II indicates that our approach gives more accurate estimation of $H_{\mathrm{B}}$ using shadow information in an automated manner. The average processing times for one building, one scene, and all seven test images were $0.10 \mathrm{~s}, 1.50 \mathrm{~min}$, and $4 \mathrm{~min}$, respectively. Execution time depends on the size of the image and the complexity of the scene, as shown in Table I. Smaller images (e.g., \#1 and \#6) with one or two rectilinear buildings require less processing time and have the lowest estimation error.

\section{Conclusion}

In this letter, we presented a novel shadow-overlapping algorithm, $A_{\mathrm{SO}}$, for estimating building heights from a single VHR multispectral image. The new approach is based on the automated identification of building shadow regions using the solar information in the image metadata, morphological operations, and Jaccard index $(J I)$. The algorithm is tested on different urban scenarios with varying building and neighborhood attributes. Results are encouraging and outperform past approaches with a $21 \%$ reduction in mean error and an overall accuracy of $\sim 80 \%$. The increased accuracy is attributed to the consideration of overlapped shadow regions and the removal of landscape features (e.g., the shadows of vegetation canopies).

The core benefit of our approach is the cost-effective extraction of building height and subsequent 3-D construction of urban areas. Applications can range from 3-D urban change monitoring to the high resolution assessment of potential and forecasting of renewable energy such as solar photovoltaics and wind. The speed and frequency (e.g., daily) of VHR acquisition compared to the more expensive methods such as LiDAR open up significant possibilities for emergency response such as the assessment of damage to buildings and infrastructure immediately after a disaster event. Future work can expand on our methodology to enhance accuracy by differentiating between the terrain and building shadows, as well as by integrating multiple methods and data sources, such as DSMs.

\section{REFERENCES}

[1] P. McKeen and A. S. Fung, "The effect of building aspect ratio on energy efficiency: A case study for multi-unit residential buildings in Canada," Buildings, vol. 4, no. 3, pp. 336-354, 2014.

[2] N. Kadhim, M. Mourshed, and M. Bray, "Advances in remote sensing applications for urban sustainability," Eur-Medit. J. Environ. Integr., vol. 1, p. 7, Dec. 2016.

[3] M. Mourshed, A. Bucchiarone, and F. Khandokar, "Smart: A processoriented methodology for resilient smart cities," in Proc. IEEE Int. Smart Cities Conf. (ISC2), Sep. 2016, pp. 1-6.

[4] J. Hu, S. You, and U. Neumann, "Approaches to large-scale urban modeling," IEEE Comput. Graph. Appl., vol. 23, no. 6, pp. 62-69, Nov. 2003.

[5] S. A. N. Gilani, M. Awrangjeb, and G. Lu, "An automatic building extraction and regularisation technique using lidar point cloud data and orthoimage," Remote Sens., vol. 8, no. 3, p. 258, 2016.

[6] N. M. S. M. Kadhim, M. Mourshed, and M. T. Bray, "Shadow detection from very high resoluton satellite image using grabcut segmentation and ratio-band algorithms," Int. Arch. Photogramm., Remote Sens. Spatial Inf. Sci., vol. 40, no. 3, pp. 95-101, 2015.

[7] T. Kim, T. Javzandulam, and T.-Y. Lee, "Semiautomatic reconstruction of building height and footprints from single satellite images," in Proc. IEEE Int. Geosci. Remote Sens. Symp., Jul. 2007, pp. $4737-4740$.

[8] T. Lee and T. Kim, "Automatic building height extraction by volumetric shadow analysis of monoscopic imagery," Int. J. Remote Sens., vol. 34, no. 16, pp. 5834-5850, 2013.

[9] M. Izadi and P. Saeedi, "Three-dimensional polygonal building model estimation from single satellite images," IEEE Trans. Geosci. Remote Sens., vol. 50, no. 6, pp. 2254-2272, Jun. 2012.

[10] Y. Shao, G. N. Taff, and S. J. Walsh, "Shadow detection and buildingheight estimation using IKONOS data," Int. J. Remote Sens., vol. 32 no. 22, pp. 6929-6944, 2011.

[11] A. Comber et al., "Using shadows in high-resolution imagery to determine building height," Remote Sens. Lett., vol. 3, no. 7, pp. 551-556, 2012.

[12] P. L. N. Raju, H. Chaudhary, and A. K. Jha, "Shadow analysis technique for extraction of building height using high resolution satellite single image and accuracy assessment," Int. Arch. Photogram., Remote Sens. Spatial Inf. Sci., vol. 40, no. 8, pp. 1185-1192, 2014.

[13] N. M. Kadhim, M. Mourshed, and M. Bray, "Automatic extraction of urban structures based on shadow information from satellite imagery," in Proc. 14th Conf. Int. Building Perform. Simulation Assoc. (IBPSA), 2015, pp. 2607-2614.

[14] F. Qi, J. Z. Zhai, and G. Dang, "Building height estimation using Google Earth," Energy Buildings, vol. 118, pp. 123-132, Apr. 2016.

[15] G. Liasis and S. Stavrou, "Satellite images analysis for shadow detection and building height estimation," ISPRS J. Photogram. Remote Sens., vol. 119, pp. 437-450, Sep. 2016.

[16] D. Rüfenacht, C. Fredembach, and S. Süsstrunk, "Automatic and accurate shadow detection using near-infrared information," IEEE Trans Pattern Anal. Mach. Intell., vol. 36, no. 8, pp. 1672-1678, Aug. 2014.

[17] H. Sturges, "The choice of a class interval," J. Amer. Statist. Assoc., pp. 65-66, 1926.

[18] N. Otsu, "A threshold selection method from gray-level histograms," Automatica, vol. 11, nos. 285-296, pp. 23-27, 1975.

[19] A. O. Ok, "Automated detection of buildings from single vhr multispectral images using shadow information and graph cuts," ISPRS J. Photogramm. Remote Sens., vol. 86, pp. 21-40, Dec. 2013. 